Elsevier required licence: (C) <2017>. This manuscript version is made available under the CC-BY-NC-ND 4.0 license http://creativecommons.org/licenses/by-nc-nd/4.0/ 


\title{
Compressive sensing for efficient health monitoring and effective damage detection of structures
}

\author{
Madhuka Jayawardhana ${ }^{1}$, Xinqun Zhu, Ranjith Liyanapathirana, Upul Gunawardana \\ School of Computing Engineering and Mathematics, \\ University of Western Sydney, \\ Locked Bag 1797, Penrith NSW 2751, Australia.
}

\begin{abstract}
Real world Structural Health Monitoring (SHM) systems consist of sensors in the scale of hundreds, each sensor generating extremely large amounts of data, often arousing the issue of the cost associated with data transfer and storage. Sensor energy is a major component included in this cost factor, especially in Wireless Sensor Networks (WSN). Data compression is one of the techniques that is being explored to mitigate the effects of these issues. In contrast to traditional data compression techniques, Compressive Sensing (CS) - a very recent development - introduces the means of accurately reproducing a signal by acquiring much less number of samples than that defined by the Nyquist's theorem. CS achieves this task by exploiting the sparsity of the signal. By the reduced amount of data samples, CS may help reduce the energy consumption and storage costs associated with SHM systems. This paper investigates CS based data acquisition in SHM, in particular, the implications of CS on damage detection and localization. CS is implemented in a simulation environment to compress structural response data from a Reinforced Concrete (RC) structure. Promising results were obtained from the compressed data reconstruction process as well as the subsequent damage identification process using the reconstructed data. A reconstruction accuracy of $99 \%$ could be achieved at a Compression Ratio (CR) of 2.48 using the experimental data. Further analysis using the reconstructed signals provided accurate damage detection and localization results using two damage detection algorithms, showing that CS has not compromised the crucial information on structural damages during the compression process.
\end{abstract}

Keywords: structural health monitoring, compressive sensing, wireless sensor networks, data compression

Email addresses: m.jayawardhana@uws.edu.au (Madhuka Jayawardhana), xinqun.zhu@uws.edu . au (Xinqun Zhu), r.liyanapathirana@uws.edu.au (Ranjith Liyanapathirana), u.gunawardana@uws.edu.au (Upul Gunawardana)

${ }^{1}$ Corresponding author 


\section{Introduction}

Transmitting and maintaining large amounts of data in SHM systems have been issues addressed in recent researches due to the large scale real-world structures in use. Traditionally, uncompressed structural response signals are acquired by each sensor and transmitted to a central server for processing and damage decision making. This task unnecessarily consumes one of the most valuable resource in a WSN-based SHM system - energy, and also wastes memory space of sensor nodes in both wired and wireless systems. In a wireless-based SHM system, such wastage results in reduced system lifetime and increased maintenance costs, since data transmission in a WSN is carried out at the cost of limited battery power of sensors. For both types of systems, transmitting a lot of data also increases the network traffic and collisions, reducing the reliability of data communication process. Even though memory is becoming cheaper, even the newest version of the most widely used commercially available sensor node - Imote2 - has only a combined memory of 64 MB. This amount of memory will barely be sufficient to a network as large as the SHM system installed on Tsing Ma, Ting Kau and Kap Shui Mun bridges in Hong Kong [1, 2]. This system, known as the "Wind And Structural Health Monitoring System" (WASHMS) consists of approximately 800 sensors and operates 24 hours a day, 7 days a week, where each sensor node measures temperature, strains in structural members, wind speed deflection and rotation of the kilometres of cables in the bridges and any movement of the bridge decks and towers, generating an enormous amount of data [3].

There have been numerous proposals to mitigate the effects of these issues including decentralized data processing in SHM [4], power saving strategies [5] and data compression techniques [6]. In traditional data compression techniques, compression is applied on the already sampled signal. However, this usually leads to wastage of processing power and memory as the important information in most signals is carried only by a few samples compared to the total number of samples. The novel concept of CS appears a potential 
alternative to solve this issue. By exploiting the sparsity of the signal, CS provides the means of accurately reconstructing the signal with much less number of acquired samples than that defined by the Nyquist's theorem [7, 8]. This concept is believed to have tremendous potential in the field of WSNs [7]. In fact, already there have been a few attempts to use this method in WSNs in the literature [9, 10, 11]. Being an application of WSN, SHM may also benefit from the compressing abilities of CS. With CS used for data acquisition in SHM systems, the energy and storage costs associated will be vastly reduced [2]. Thus, CS can be an area with high potential for research and development in the field of SHM. A preliminary study on the feasibility of adopting CS for SHM was presented in [12].

In this paper, CS based data acquisition in SHM is explored, in particular for damage detection and localization. The attempt is to preserve the energy and storage associated with SHM systems through data reduction, without losing the confidence of accurate decisionmaking. The effectiveness of CS for data compression in SHM is analyzed using a set of experimental data from a series of impact tests carried out on an RC slab. Promising results were obtained from this application in terms of CRs and reconstruction accuracy. Further analyses on the reconstructed signal using two damage detection and localization algorithms (the ARD method [13] and the Wiener filter based method [14, 15]) provided successful damage detection and localization results. The main contributions of this paper are as follows:

- Proposing the use of CS based data acquisition for reliable SHM.

- Successful reconstruction of the compressed responses.

- Comparison of the data compression performance using CS and other compression techniques.

- Successful damage detection and localization using the reconstructed responses. 
The remainder of this paper is organized as follows: First, the current work in literature on the applications of CS are discussed. Then the theory of CS is presented followed by the application of CS on the available experimental data. The success of the CS based data acquisition is analyzed using the achieved CRs and the accuracy of signal reconstruction in terms of a frequency content based metric that was developed in this study. Next, a brief comparison is carried out between two other data compression techniques and CS. Finally, damage detection and localization results using the CS reconstructed data are presented followed by the conclusions.

\section{Related work}

$\mathrm{CS}$ is a quite recent development in communication and data processing technologies which has been recently introduced to WSNs. CS has the potential to serve as alternatives to both Nyquists rate sampling and data compression techniques. Being a novel concept, applications of CS are largely under-explored in the field of sensor networks. Only a limited number of studies exist on CS in SHM applications in the literature. Bajwa et al. introduced the concept of CS for WSNs [9]. This work discusses the power-distortion trade-off associated with the data acquisition through CS in a centralized WSN, and the latency involved in the information retrieval. Then, a distributed matched source-channel communication scheme is proposed for the estimation of sensed data at the fusion center or the central server. Feng et al. investigate the problem of sensor localization in WSNs and proposes a novel method called Compressive Sensing for Manifold Learning [10]. The measurement matrix for data acquisition is chosen to be the pair-wise distance matrix on which CS is applied. This pair-wise measurement matrix is constructed by each node measuring the distance to all its neighbouring nodes. A central node retrieves the compressive measurements and reconstructs the full matrix in order to construct the location map of the sensors. This work shows the high accuracy of the achieved localization results even though the distance 
measurement matrix is reconstructed at the central node with only a limited number of samples from the original measurement. The communication cost reduction associated with the method is also proven. A distributed algorithm for sparse signal recovery is introduced by Ling and Tian [11. While a fraction of the sensors are turned off by a sleeping strategy, compressive sampling is performed on the awake sensors. These sensors collaborate with their active neighbours through one-hop communication to recover the compressed measurements and iteratively improves the local estimates until reaching a global maximum. The performance of this algorithm is illustrated in terms of scalability and optimal data acquisition.

Cortial et al. discuss the application of CS in structural damage detection [16]. They demonstrate the effectiveness of the application by using CS data to successfully locate the damage in a F-16 fighter jet simulation. Bao et al. investigate the performance of CS for compression of vibration data obtained from an actual SHM system [2, 17]. Reconstruction of the signal is done using both wavelet and Fourier orthogonal bases. Their results show that the CRs achieved using CS in this case are not very high. They account this poor result to the fact that the vibration data of civil structures used for SHM are not naturally sparse in the chosen bases. In their later work Bao et al. apply CS to recover lost data in a WSN used in SHM. Their results show that the signal recovery is good when the signal is sparse regardless of continuous or random packet loss and noise, demonstrating the significance of sparsity for CS [18]. Wang and Hao were the first to propose a damage identification scheme based on CS for SHM [19]. Mapping damage identification as pattern classification problems, they construct a feature matrix based on the sparse representation results of time domain structural responses. They demonstrate the success of the method using numerical and experimental data. However, the application of this method to complex large civil structures could be a challenging task as the time taken to train the model on damage cases may be too high. Mascarenas et al. address the challenges of extracting information 
on damage condition from large heterogeneous sets of data from structures and the costeffective collection of relevant data, respecting the limitations of the system in their work [20]. They propose a two-stage CS framework with potential to solve both these challenges. They evaluate the implementation of the compression filter named smashed filter for damage classification in the structure with successful results. Yang and Nagarajaiah present a novel algorithm for both locating and assessing structural damage using Blind Source Separation (BSS), sparse representation and CS [21]. In this algorithm they first extract modal features of the structure blindly using an unsupervised Complexity Pursuit (CP) algorithm and then use the sparse nature of the classification network established based on CS to classify damage. In a later study they work on denoising of structural responses through a new technique named Principal Component Pursuit (PCP) which is inspired by CS [22]. In [23] they propose a new method output-only modal identification of structures using a combination of BSS and CS and show that the method can accurately identify the modes using low-rate sampling than that required by the Nyquist theorem.

As the theory states, when applied appropriately, CS can be expected to acquire undersampled structural responses from sensors in an SHM system and accurately reconstruct them at the central server. This will save sensor energy spent on data transmission, save sensor storage space and data maintenance costs by reducing the amount of data, while still enabling accurate reconstruction of the data providing the ability to process the data at convenience. This overall picture, as well as the successful application of CS in recent researches in communication and networking inspired the application of CS for SHM.

\section{Compressive Sensing for Structural Health Monitoring}

Traditional sampling exploits the band-limitedness of a signal, sampling it at twice (or more) as fast as its bandwidth (the Nyquist rate), to ensure accurate reconstruction [24]. CS exploits the sparsity of a signal during its sampling [7]. The concept of CS [2, 7, 9, 8] is 
summarized in the following section using this principle of sparsity.

\subsection{Compressive sensing}

The applicability of CS for successful data compression can be explained with the aid of two principles: Sparsity and Incoherence, which are important in the understanding of the theory of CS.

SPARSITY: Many natural and man-made signals are sparse or compressible in the sense that, there exists a base $\Psi$ where the representation in Equation (1) has just a few large coefficients and many small coefficients. In other words, they have a concise representation when expressed in an appropriate basis $\Psi$. Another word to explain this situation is redundancy of a signal when expressed in an appropriate basis.

INCOHERENCE: This states the idea that a signal having a sparse representation in $\Psi$, must be spread out in the domain in which they are acquired. An example is the impulse response in the time domain, which spreads out in the frequency domain.

A real-valued, finite length, one-dimensional, discrete time signal can be expressed as $x(t), t=1,2, \ldots, n$ in $\Re$ where $n$ is the signal length. In this study, $x(t)$ is the data to be compressed (e.g. acceleration, strain) and can be represented in terms of a basis of $n \times 1$ vectors $\psi_{i}, i=1,2, \ldots, n$. For convenience, the basis is assumed to be orthonormal. Some Wavelet families, Fourier basis and cosine transforms are examples for possible bases for the data. The $n \times n$ basis matrix $\Psi=\left[\psi_{1}, \psi_{2}, \ldots, \psi_{n}\right]$ is then formed by arranging the vectors $\psi_{i}$ as columns. With $\Psi^{-1}=\Psi^{T}$ and the vector $x=[x(1), x(2), \ldots, x(n)]^{T} \in \Re^{n}, n$ number of sampled values of $x(t)$ can be expressed as:

$$
x=\sum_{j=1}^{n} \alpha_{j} \psi_{j} \quad \text { or } \quad x=\Psi \alpha,
$$

where, the basis coefficients $\alpha_{j}$ are given by $\alpha=\Psi^{T} x$. 
By a linear projection of $x$, a vector $y$ of length $m(<n)$ can be acquired as follows:

$$
y=\Phi x=\Phi \Psi \alpha=\Theta \alpha,
$$

where, $\Phi$ is the $m \times n$ measurement matrix and $\Theta=\Phi \Psi$ is an $m \times n$ matrix.

Since $m<n$ in $\Phi$, recovering the original response $x$ of length $n$ from the $m$ measurements in $y$ is ill-posed. If the signal $x$ is known to be sparse in the basis $\Psi$, i.e., many of the coefficients in $\alpha$ are zero, then under certain conditions, $\alpha$ can be reconstructed exactly by solving the following convex optimization:

$$
\hat{\alpha}=\arg \min \|\tilde{\alpha}\|_{1} \quad \text { such that, } \quad \Theta \tilde{\alpha}=y .
$$

where, $\|\tilde{\alpha}\|_{1}$ is the $l_{1}$ norm of $\tilde{\alpha}$.

Given that; 1) the signal $x$ is $s$-sparse (it has at most $s$ non-zero entries) and 2) the matrix $\Theta$ obeys the Restricted Isometric Property (RIP) described below, $\alpha$ can be precisely recovered by solving Equation (3).

The RIP [8] states that, there exists an isometric constant $\delta_{s}$ for the matrix $\Theta$, where $\delta_{s}$ is defined as the smallest number such that for all $s$-sparse vectors $v_{s}$, the following condition holds:

$$
\left(1-\delta_{s}\right)\left\|v_{s}\right\|_{2}^{2} \leqslant\left\|\Theta v_{s}\right\|_{2}^{2} \leqslant\left(1+\delta_{s}\right)\left\|v_{s}\right\|_{2}^{2}
$$

Therefore, the $s$-sparse signals can be exactly recovered by solving the optimization in Equation (3), if $\Theta$ satisfies Equation (4). In other words, suppose that $\Psi$ is an orthonormal basis (for simplicity) such as a wavelet basis or a Fourier sinusoids basis. Then, a measurement matrix $\Phi$ needs to be constructed such that, the matrix $\Theta(\Theta=\Phi \Psi)$ obeys Equation (4).

In $\mathrm{CS}, \Phi$ is selected as a random $m \times n$ matrix, such as: 
1. by sampling independent and identically distributed (i.i.d.) entries $\phi_{i, j}$ from a normal distribution with zero mean and $1 / m$ variance

2. by sampling i.i.d. entries $\phi_{i, j}$ from a symmetric Bernoulli distribution $\left(P\left(\phi_{i, j}=1 / m\right)\right)$

3. by sampling $n$ column vectors uniformly at random on the unit sphere of $\Re^{m}$

4. by sampling a random projection $P$ and normalizing; $\Phi=n / m P$

The matrix $\Phi$ obtained from either of the above methods obeys the RIP with overwhelming probability given the following condition is fulfilled [7].

$$
m \geqslant \frac{c s}{\log _{10}(n / s)}
$$

where, $c$ is a specified constant depending on each instance.

When the above condition is satisfied, the matrix $\Theta$ also obeys the RIP with $m \geq$ $c s / \log _{10}(n / s)$ regardless of the orthonormal basis matrix $\Psi$. And it also becomes incoherent with $\Psi$. Therefore, the perfect reconstruction of an $s$-sparse response signal $x$ of length $n$ is possible independently of its basis $\Psi$, if the ratio $n / m$ does not exceed $c^{-1}(n / s) \log _{10}(n / s)$. This makes $n / m$ a measure of sparsity of the signal $x$ [2].

As a summary, the ultimate aim is to obtain a compressed signal $y$ through a linear projection of $x$ as shown in Equation (2), and be able to accurately reconstruct $x$ by solving the convex optimization in Equation (3). In CS, this is rendered possible by selecting the measurement matrix as described above, given that the constraint in Equation (5) is fulfilled. Then, the original signal $x$ can be reconstructed using the measured signal $y$ by solving the convex optimization given in Equation (3).

\subsection{Compressing noisy signals}

When responses are contaminated with noise, those signals may not be naturally sparse in any basis. Therefore, de-noising the response is required to make the signal sparse. In 
such a case, the measured signal $y$ can be represented as follows:

$$
y=\Phi x+e,
$$

where, $e$ is the error associated with noise, which is uncertain and bounded as $\|e\|_{2} \leqslant \epsilon$ with $\epsilon$ being a chosen bound on the size of the measurement error.

The basis coefficients $\alpha$ can then be reconstructed by solving the following convex optimization:

$$
\hat{\alpha}=\arg \min \|\tilde{\alpha}\|_{1} \quad \text { such that }\|\Theta \tilde{\alpha}-y\|_{2} \leqslant \epsilon,
$$

where, $\mid \Theta \tilde{\alpha}-y \|_{2}$ is the $l_{2}$ norm of $\Theta \tilde{\alpha}-y$.

Similar to the optimization in Equation (3), Equation (7) also has a unique solution for $\hat{\alpha}$ which can be used to represent the data. But in this case, since the unknown noise is only assumed to be bound on its norm, the exact recovery of the original response $x$ cannot be guaranteed [2]. Having discussed the theory of CS, the focus will now be on adopting CS for data acquisition in SHM systems, which will be discussed in the following sections.

\section{Experimental evaluation}

In order to verify the effectiveness and accuracy of the process, a set of experimental data is used from a series of experiments carried out on an RC slab. These data will be briefly described in this section before proceeding to the details of CS application: CS requirements and how the data fits with the requirements, data compression with CS and analysis of the reconstruction. The simulation work in this paper has been carried out using MATLAB.

\subsection{Experimental setup}

A two span RC slab has been used as the test specimen in this experiment [25]. Each span of the slab was loaded with a two-point loading system as shown in Figure 1a, which 
was also connected to the supports in order to reduce the effect on the supports. Damages were created on the slab by incrementally loading it via a static load test in 13 loading levels while monitoring damage locations and lengths. A dynamic load test was carried out on the slab using an impact hammer, in order to record structural responses. The slab was equipped with nine sensors evenly distributed along the length of the slab on its central axis. A data acquisition system was used to acquire six acceleration measurements at each loading stage at $500 \mathrm{~Hz}$ sampling rate giving six test samples of structural responses, each of length 4100. Figure $1 \mathrm{~b}$ illustrates the crack damages created on the slab and their propagation along the slab with increasing levels of static loading. These experiment records show three main areas of damage starting from the right span propagating through the middle support area to the left span. These damage areas are identified as damage zones for the purposes of this paper. A sample measurement and its frequency spectrum obtained using Fast Fourier Transform (FFT) is illustrated in Figure 2 .

\subsection{Compressive sensing pre-requisites}

For feasible reconstruction of the signal, CS requires the data to be compressed, to be sparse in some basis. Therefore, the first step in adopting CS for data acquisition in SHM systems is to analyze the experimental data for their sparsity. Several commonly used methods such as the wavelet transform, Fourier transform and Discrete Cosine Transform (DCT) were initially applied on the structural responses. However, the responses exhibited no sparsity in either of the above bases. This can be further examined looking at the zoomed portion of the signal - from data point 3000 to 3050, in the 4100 length original sample shown in Figure 2a. It can be seen in this figure that although the magnitude of the signal appears to be zero in the latter part of the sample, it always has a negligibly small but non-zero value. Nevertheless, looking at the signal, it can be reasonably considered that most of the information in this structural response is carried by only a few data points. In other words, most of the data points appear to be negligibly small such as noise. Therefore, de-noising 
techniques were adopted in order to make the signal sparse in some basis.

Wavelet de-noising technique was selected to de-noise the signal in this case due to its simplicity and versatility [26, 27]. Wavelet de-noising is also the most common method used for signal de-noising [26, 27]. There are two main de-noising techniques used in wavelet denoising: Soft thresholding and Hard thresholding. With Soft thresholding, the signal points are either shrinked to fit the selected threshold or killed (made '0'). In Hard thresholding, the signal points are either retained as it is or killed [2, 26, 27].

Several methods exist for the determination of the threshold in the de-noising process, such as the principle of Stein's unbiased Risk Estimator, Minimax thresholding and Universal threshold [28, 29]. MATLAB offers four methods for selecting threshold: a) Stein's Unbiased Risk (rigrsure), b) Heuristic variant of the Stein's technique (heursure), c) Universal threshold (sqtwolog), d) Minimax threshold (minimaxi).

Due to its compression abilities and also because of its popular use in CS applications in the literature [2, 7, 8], wavelets were selected as the preferred basis functions for this study. Further, Daubechies wavelet family was selected due to its sophistication compared to other wavelet families in use [30]. Figure 3 shows a comparison of the original signal and the de-noised signals with different threshold selection methods indicated above. These results were obtained using Hard-thresholding, which is a choice that will be justified later in the section. It should be noted that although the complete signal of length 4100 is used in all the actual analyses throughout this study, only the first half of it that contains the impulse response (a length of 2050) is plotted in most of the graphs included in this paper for better clarity.

The de-noised signals using the above mentioned threshold selection methods show following sparsities (number of non-zero points) in the Daubechies wavelet basis: 1) rigrsure, $s$ $=110,2)$ heursure, $s=23,3)$ sqtwolog, $s=23,4)$ minimaxi, $s=40$. The de-noised signals using these threshold selections are illustrated in the last four plots of Figure 3 compared 
with the original signal in the first plot. It is evident from Figure 3 that de-noising has considerably reduced the noise associated with the signal. From this analysis, Stein's Unbiased Risk method is chosen for threshold estimation as it gives the highest sparsity value above (rigrsure with $s=110$ ). This is also visible in the Figure 3 , where the rigrsure figure is the closest representation of the original signal shown in the first figure, as opposed to the rest of the de-noised figures. The reason for this choice is that for post-processing work such as damage detection, as much information as possible from the original signal has to be retained through the compression process.

Figure 4 shows a comparison of the hard-thresholding and soft-thresholding techniques compared with the original signal. The Stein's Unbiased Risk method for threshold estimation as chosen previously, was used in this analysis. It can be seen from this figure how Hard thresholding retains more information than Soft thresholding. Since in this application the actual information of the signal should be retained as much as possible without shrinking for post-processing purposes such as damage detection and localization, Hard thresholding technique was selected for the wavelet de-noising process. With knowledge of the signal sparsity from the de-noising process, compression of the signal can now be carried out. The results of the compression and the reconstruction processes will be discussed in the next section.

\subsection{Signal compression and reconstruction}

As the de-noised structural response using Stein's Unbiased Risk threshold selection and Hard thresholding technique exhibited sparsity in the Daubechies wavelet basis, accurate signal reconstruction should now be feasible. However, due to the noise term involved in the signal, the measurement signal to be acquired in the current scenario resembles Equation (6) and the convex optimisation problem to be solved is of the form shown in Equation (7). The bound on the measurement noise $\epsilon$ is ideally estimated by taking the norm of the noise measured either using a sensor that is not programmed for CS or by prior knowledge. In 
this study, it was initially estimated by optimizing the accuracy of reconstruction (discussed later in this section). However, the reconstruction quality of the signal was found to be independent of this choice in the current analysis which simplifies the situation, as knowledge of the complete signal would not be available in a real-world CS scenario.

When the sparsity of the de-noised signal is known, the measurement matrix $\Phi$ of Equation (6) can be selected. As mentioned in Section 3, in CS, this measurement matrix is required to be random in order to accurately reconstruct the compressive sampled signal. It also needs to fulfil the condition in Equation (5) in order to satisfy the RIP in Equation (4), again for accurate reconstruction of the signal. Therefore, as a start, the length of the measurement sample $m$ was chosen to be twice the value of sparsity ( $m=2 \times s$ ) fulfilling the condition in Equation (5). The $m \times n$ measurement matrix is obtained by sampling i.i.d. entries from a normal distribution with zero mean and $1 / m$ variance.

Using the sparsity of 110 from the chosen de-noising technique, the compressed length becomes $m=220$. CR is defined as the ratio between the lengths of the original (uncompressed) signal and the compressed signal. The CR of the current scenario is $n / m=18.64$. Subsequently, compression of the signals with $m=3 \times s, m=4 \times s, m=5 \times s, m=10 \times s$ and $m=15 \times s$ will also be examined pertaining to the constraint defined by Equation (5), resulting in CRs of 12.42,9.32,7.45,3.73 and 2.48. Figure 5 illustrates the compressed signals at these six compression levels. In the framework of this study, the compressed signal is obtained by a linear projection of the original data using the chosen measurement matrix, since the original data is already available. However, in an actual system, the signal needs to be obtained directly in the compressed form at sampling. For this, the measurement matrix needs to be built into the sensor before sensor installation, which is not discussed in this study. It can be seen from Figure 5 that as the compressed length increases, more information from the original sample is retained in the compressed signal at the cost of CR.

At reconstruction, the receiver or the central server in case of an SHM system, has 
knowledge of the utilized measurement matrix and the sparse basis. In order to solve the modified convex optimization problem in Equation (7), CVX, a package built on MATLAB for specifying and solving convex problems [31] is utilized.

Figure 6 illustrates the reconstructed structural responses from compressed signals $m=$ $2 \times s, m=3 \times s, m=4 \times s, m=5 \times s, m=10 \times s$ and $m=15 \times s$, and their frequency spectra. Once again, only the first half of the signal is plotted here for clarity. It can be seen in this figure that the reconstruction improves with increasing compressed sample length. That is, as the CR decreases, the reconstructed signal better resembles the original signal, as can be expected. A similar variation can be seen with the frequency spectra. From these results, reconstruction from the compressed signal of length $m=15 \times s$ (CR of 2.48) resembles the original signal best. It can be predicted from this trend that, with an increased number of compressed samples (within the constraints of Equation (5)) the quality of the reconstructed signal will improve with better resemblance to the original signal. However, this will reduce the CR, thereby reducing the advantage provided by the compression. Therefore, the CR and the reconstruction quality presents a trade-off.

Analyzing the accuracy of the reconstruction is vital when validating a data compression technique, as the important information carried by the signal should not be compromised by the compression process. Especially in SHM measurements, retaining the frequency content of the measurements is crucial as they carry important information required for structural condition analysis in the data post-processing. Therefore, a reconstruction accuracy metric $R A_{C S}$ to quantify the accuracy of the reconstructed signal in this analysis is computed as follows:

$$
R A_{C S}=\operatorname{corrcoe} f\left(\left|\mathrm{fft}\left(x_{\text {Orig }}\right)\right|,\left|\mathrm{fft}\left(x_{\text {CSrecon }}\right)\right|\right),
$$

where, $x_{\text {Orig }}$ is the original signal and $x_{C \text { Srecon }}$ is the CS reconstructed signal with 'fft' 
indicating the fast Fourier transform.

$$
\operatorname{corrcoef}(x, y)=\frac{\sum_{k=1}^{M}\left(x(k)-\mu_{x}\right) \cdot\left(y(k)-\mu_{y}\right)}{\left\|x-\mu_{x}\right\| \cdot\left\|y-\mu_{y}\right\|},
$$

is the correlation coefficient of data samples $x$ and $y$, where $M$ is the data length with $k=1,2, . ., M$ and $\mu_{x}$ and $\mu_{y}$ are the means of $x$ and $y$ respectively.

Figure 7 shows the reconstruction accuracy $R A_{C S}$ of the reconstructed signals from Figure 6, compared to a reference $R A_{C S}$ value of ' 1 '. This reference value $R A_{C S}=1$ results from a perfect signal compression-reconstruction process where the reconstructed signal matches the original signal perfectly, and is obtained by comparing the reconstructed signal with itself. In Figure 7 , the $R A_{C S}$ values of the reconstructed signals from different compressed signals lengths are plotted. It is clear from this figure that with an accuracy with $R A_{C S}=0.9777$, reconstruction from the sample of length $10 \times s(C R=3.73)$ is a very good representation of the original signal, and the15 $\times s(C R=2.48)$ signal reconstruction is even more so with an almost perfect accuracy with $R A_{C S}=0.9923$.

Signal compression and reconstruction with varying de-noising thresholds are analyzed next in order to observe the effects of the threshold estimation methods on the CS process. The threshold estimation criteria discussed previously, a) rigrsure, b) heursure, c) sqtwolog and d) minimaxi are considered in this analysis. Figure 8 illustrates the compressed samples obtained by compressing the de-noised data. It can be seen how the Stein's Unbiased Risk (rigrsure) has more length - and thereby more information - compared to the other three techniques. A compressed length of $10 \times s$ which depends on the sparsity $(s)$ of the de-noised signals from each of the above threshold selections is used in this analysis.

The corresponding reconstructed data and their frequency spectra are shown in Figure9. As with the earlier figure of the reconstructed data, only the first half of the signal is plotted for better clarity of the graph. It can be seen how the Stein's Unbiased Risk (rigrsure) 
thresholding enables better reconstruction compared to the other three methods. A similar observation can be gained from the frequency spectra, where the reconstruction from Stein's Unbiased Risk method resembles the original signal's spectrum better than the rest. Further, out of the other three methods, Minimax threshold (minimaxi) has better results compared to the Heuristic variant of the Stein's technique (heursure) and the Universal threshold (sqtwolog) methods. Figure 10 illustrates the reconstruction accuracy results of this analysis using the $R A_{C S}$ given in Equation (8). Compared to the reference value $R A_{C S}=1$ resulting from a perfect reconstruction, Stein's Unbiased Risk method (rigrsure) has shown a very good accuracy with $R A_{C S}=0.9777$. The Minimax threshold (minimaxi) has also shown a reasonable accuracy with $R A_{C S}=0.8609$.

Further into the analysis, Figure 11 shows the combined CS-reconstruction accuracy results from the two previous discussions. The $R A_{C S}$ is plotted against the de-noising sample length (or the CR), and the four different threshold estimation methods. It can be seen that each of the de-noising methods exhibit similar results with varying CR. That is, as the compressed length increases, de-noised signals from all four threshold estimation methods can be reconstructed with better accuracy at the cost of CR. Stein's Unbiased Risk (rigrsure) method for threshold estimation proves to be the most effective in this process in terms of the reconstruction accuracy.

Nevertheless, as the most essential task required by an SHM system is monitoring and deciding on the structural condition, the data available for post-processing and decisionmaking should be reliable enough. Therefore, if the important information embedded in the original signal is not lost in the process of compression and reconstruction, the compression technique can be considered as good enough to be employed in an SHM system for decision making. This possibility will be explored using damage detection and localization algorithms in Section 5 . 


\subsection{Sensor energy and memory space saving with compressive sensing}

Energy is not an issue for a wired sensor network as the sensors could have a never ending supply of power through the connecting cables. However, limited energy of wireless sensors is a major issue which has attracted the attention of researchers worldwide. Research is still on-going for a sustainable solution to this problem. Limited sensor memory space can be an issue to both these network types. However, since the sensors could be programmed to transmit the data continuously to the central server in a wired network, sensor memory space can be freed often, making its impact somewhat less in a wired network. Nevertheless, the cost associated with data transmission and management is a problem for any such network. Hence, a discussion on the ability of CS for sensor energy and memory saving is presented in this section.

It is said that transmitting one bit through wireless communication may consume as much energy as executing a few thousand instructions in a processor [32, 33]. It is clear that the transmission energy increases with increasing data amount to be transmitted. As defined previously, $\mathrm{CR}$ is the ratio between the original signal length and the compressed signal length. It is a fact that some control information are added to a data packet before it is transmitted to the central server. However, compared to a data sample of length 4100 as with this analysis, CR could be considered a good indicator of the energy savings in the data transmission process. For sensor memory saving, CR is an obvious indicator. Therefore, for the purpose of this discussion, CR is used as an approximate indicator of both sensor energy and memory saved in the CS process.

Figure 12 shows how the CR changes - and hence the sensor energy and memory savings change - with varying compressed data sample lengths and CS reconstruction accuracies. The $x$-axis represents length of the compressed data sample in terms of the sparsity of the signal $s$, similar to the previous discussions. The fist $y$-axis on the left hand side of the plot represents the sensor energy/memory saving with respect to the energy/memory consumed 
by the uncompressed signal. This is presented in terms of the ratio between the energies consumed (ex: 20* in Figure 12 means 20 times the energy consumed by the original signal). The second $y$-axis on the right hand side represents the CS reconstruction accuracy of the signal in terms of the $R A_{C S}$ metric introduced in the previous section. As can be seen in the figure, the $R A_{C S}$ increases with the increasing compressed length which was already observed and discussed in a previous section. The sensor energy/memory saving decreases with increasing compressed sample length as can be expected. When the compressed length is $15 \times s$ with an almost perfect reconstruction of the original signal $\left(R A_{C S}=0.9923\right.$, Figure 7), CS still achieves an energy/memory saving of approximately $2.5(\mathrm{CR}=2.48)$. In other words, more than $50 \%$ of the energy/memory is saved in this scenario compared to the uncompressed signal. In all the other compressed lengths shown in the figure, more energy/memory is saved than the above example. This is however, at the cost of accuracy of reconstruction. For example, at compressed length $2 \times s$, the energy/memory saving is nearly 19 times the uncompressed signal transmission $(\mathrm{CR}=18.64)$, which is approximately a $95 \%$ energy/memory saving. But the CS reconstruction is poor at this point with $R A_{C S}=0.7025$ (Figure 7).

Although the energy/memory saving decreases with increasing compressed sample length, in this study, CS has been able to save more than $50 \%$ sensor energy/memory with an impressive reconstruction accuracy of $99 \%$ at $\mathrm{CR}=2.48$. Thus, the application of CS for compressed data sampling in SHM systems shows promise in saving energy and memory space in sensors.

\subsection{Comparison with other compression techniques}

The main advantage of using CS for data compression is that in CS, the data is acquired in an already compressed form right at the moment of sampling, eliminating the need to first sample the signal at a higher rate, store it in the sensor and then process it for compression. This saves sensor energy for sampling and processing as well as sensor memory for data storage. As mentioned previously, the energy spent on data transmission by a wireless sensor 
is much more than that spent on data processing [32, 33]. Therefore, the CR that can be achieved by a compression method is also an important deciding factor. In this section, the performance of CS for data compression and reconstruction are compared against a few other commonly used compression techniques. For this analysis, the basic method of wavelet compression is chosen using different wavelet families and levels. Wavelet compression is a widely used technique for image compression [34]. In this analysis the signal is denoised and wavelet decomposed at different levels using different wavelets. Then the decomposed signal compression and compared against the original signa

The CR and the CS-reconstruction accuracy $R A_{C S}$ for the compression techniques being compared are tabulated in Table 1. In this case for representing the results we have chosen Symlet2 wavelet decomposition at level 2 and Daubechies2 wavelet decomposition at level 10 which resulted in the best CR of all wavelet types and decomposition levels that were evaluated. In terms of the CR, CS shows competitive results against the two wavelet compression techniques as tabulated. Both wavelet techniques have achieved CRs in the same league as $10 \times s$ signals in CS. However, the reconstruction accuracies of both wavelet methods are one which indicates perfect reconstruction whereas CS only give a near-perfect reconstruction with $R A_{C S}$ is $0.9 @$ erefore, it can be concluded from these results that the traditional compression techniques have performed better in terms of reconstruction accuracy while giving slightly less CR compared to CS.

In addition to the quantitative analysis above, a visual comparison of the reconstruction quality of the two wavelet compression techniques against the original signal are illustrated in Figure 10 t shows the reconstructed signals and their frequency spectra. This figure shows that the reconstructed signals from the two wavelet techniques are almost the same (if not exactly the same) as the original signal. These results show that traditional lossless compression techniques can achieve equal or better signal reconstruction accuracy than CS under same conditions. The advantage in CS only comes in the case when exact or 
near perfect reconstruction can be achieved, it will save sensor memory and energy compared to traditional compression techniques, as in CS the samples are already achieved in a compressed manner.

\section{Damage detection and localization}

The main purpose of a real-world SHM system is accurate detection and localization of damages in the structure. Achieving both of these tasks with sufficient accuracy has been a challenge faced by researchers for many years [4]. Accomplishing such a task with a reconstructed signal from a set of under-sampled data can be even more difficult. This section focuses on achieving these tasks - i.e. accurate damage detection and localization using the reconstructed data from under-sampled CS data. This analysis uses the de-noised data from Stein's Unbiased Risk threshold selection with Hard thresholding and a CR of 3.73.

Two damage detection and localization techniques are used in this analysis: 1) ARD method [13], which uses time-series models estimated from the structural responses, 2) Wiener filter based method, from the work described in [14, 15].

The ARD method is a statistical time-series based structural damage detection method which makes use of the Auto-Regressive (AR) model [13]. In this method, the structural response data is first fitted to an AR model with a suitable model order. The AR coefficients from this model are used in the damage detection process. A damage detection index called $D$ index computed using the undamaged data's AR coefficients and the current data's AR coefficients is used for damage detection. This D index is represented as:

$$
D=\sum_{i=1}^{p}\left(\phi_{i}^{y}-\phi_{i}^{x}\right)^{2}
$$

where, $p$ is the AR model order and $\phi_{i}^{y}$ and $\phi_{i}^{x}$ are the $i^{\text {th }}$ AR model coefficients of the current 
data $y$ (from unknown state of the structure) and reference data $x$, respectively. The AR model order $p$, is determined by exploring the autocorrelation function of the model residual errors [35, 36].

For damage localization, Fisher criterion $f$ is computed using the above $\mathrm{D}$ indexes for each sensor as follows:

$$
f=\frac{\left(\mu_{\text {curr }}-\mu_{\text {ref }}\right)^{2}}{\sigma_{\text {curr }}^{2}+\sigma_{\text {ref }}^{2}},
$$

where, $\mu_{\text {curr }}$ and $\mu_{\text {ref }}$ are the means of D indexes of the current (online) data and of the reference data respectively. $\sigma_{\text {curr }}^{2}$ and $\sigma_{\text {ref }}^{2}$ are the variances of the same.

The second method, which is based on the Wiener filter, determines the optimum Wiener filter for the structural response in question, and uses the Mean Square Error (MSE) of the filter coefficients as its Damage Sensitive Feature (DSF). MSE values computed from the unknown structural responses are averaged and compared against the respective reference value for damage detection. This DSF is represented in terms of the error of the optimum Wiener filter $e(n)$ as follows:

$$
\text { Wiener filter } M S E=E\left[e^{2}(n)\right] \text {, }
$$

where $e(n)=d(n)-y(n)$ with $d(n)$ being an estimation of the desired signal expected from the Wiener filter and $y(n)$ being the resulting noise-reduced filter output which is targeted to be an estimation of $d(n)$, respectively.

In the Wiener filter method, the damage localization is carried out at sensor pair level utilizing the spatial information associated with the sensors unlike in the ARD method. Cross-correlation function $(\mathrm{CCF})$ coefficients are computed from the Wiener filter MSEs of a sensor pair, and the variance of these coefficients is used as the damage location indicator. 


\subsection{Damage detection}

Figure 14 illustrates the damage detection results using the ARD method. Due to the similarity of the results of all sensors, the results of only one sensor are presented here. The mean of the $D$ indexes computed from structural responses of three loading stages representing the three damage scenarios shown in Figure $1 \mathrm{~b}$ of the experiment records described in Section 4.1 are plotted against the damage scenario. Similar damage detection results obtained from the original data (without CS) are also plotted here for reference (Figure 14a). Considering the CS-reconstructed data in Figure $14 \mathrm{~b}$, the mean $D$ indexes from the damaged structure in all three damaged scenarios show a clear increase compared to the reference mean $D$ index. This marks successful positive damage detection in all three cases. Compared with the results from the original data in Figure 14 a, $D$ indexes from the CSreconstructed data show a loss in magnitude. This can be due to the information lost during the data de-noising process, which shows the implications of the practical situation in contrast to an ideal set of data. However, it is evident that equally-good and clear damage detection results have been achieved using CS-reconstructed data.

Figure 15 shows the damage detection results of the Wiener filter method using original data and the CS-reconstructed data. As with the ARD method, presented here are only the results from one sensor due to the similarity shown by all other sensors in their damage detection results. The mean value of the Wiener filter MSE of the reference structure as well as the damaged structure are plotted in Figure 15. Three mean-MSE values representing the three damage scenarios in Figure 1b, show a clear difference from the reference mean-MSE value in Figure 15b. This indicates successful damage detection in all three cases using the CS-reconstructed data. Further, damage detection results of the same sensor using the original data illustrated in Figure 15 a shows that CS-reconstructed data can be relied-upon for equally accurate damage detection. Similar to the ARD comparison, the magnitude reduction of the Wiener filter MSE values of CS-reconstructed data compared to those of 
the original data can be a consequence of information loss during the wavelet de-nosing process.

\subsection{Damage localization}

Figure 16 illustrates the damage localization results from the ARD method. The computed Fisher criterion is plotted against the sensor number for damage localization. Figure 16a, Figure 16b and Figure 16c show the localization results of One damage zone, Two damage zones and Three damage zones respectively as per the experiment records in Figure 1b. Figure 16a has located damage in Sensor 8 agreeing with the experimental records that indicate crack damages in the right span of the structure where Sensors 6 to 8 are located (Figure 1b). Sensors 5 to 8 indicate damage in Figure $16 \mathrm{~b}$ which represents Two damage zones localization results. In experiment records of two damage zones, the right span and the mid support area of the structure where Sensors 6 to 8 and Sensors 4 to 6 are located respectively, are damaged (Figure 1b). Therefore, the localization of damage in Sensors 5 to 8 in Figure $16 \mathrm{~b}$ can be considered as accurate in localizing the two damage zones compared with the experiment records in Figure 1b. Similarly, three damage zones are accurately located in Figure 16c, most prominently by Sensors 8, 5 and 1, in right span, mid support area and left span of the structure, respectively. Therefore, comparing with the experimental records, it is evident that the damage locations have been accurately detected using the CS-reconstructed data in all three damage scenarios using the ARD method.

Figure 17 illustrates the damage localization results of the three damages cases: One damage zone, Two damage zones and Three damage zones in Figure 17a, Figure 17b and Figure 17k respectively. The normalized CCF coefficient-variance of the Wiener filter MSE values of sensor pairs are plotted against the sensor pair number. In this study, the sensors are paired with their adjacent sensors due to the linearity of the structure (Figure 1a). All sensors apart from Sensors 1 and 9 are paired with both its adjacent sensors (ex: Sensors 1-2, 2-3, 3-4 etc.), in order to accommodate as much spatial information as possible, making 
8 pairs of sensors altogether. Figure $17 \mathrm{a}$, Figure $17 \mathrm{~b}$ and Figure $17 \mathrm{c}$ have successfully located: one damage zone in right span of the structure; two damage zones in right span and mid-support of the structure; and finally three damage zones in right and left spans and mid-support of the structure respectively. Therefore, it is evident from Figure 17 that the method has successfully located damage in all three cases using the CS-reconstructed data.

Although the results of only three loading stages are presented here due to limited space, all 13 loading stages of the experiment showed accurate damage detection and localization results. The above results confirm that the ARD method and the Wiener filter method have successfully detected and located damages in the structure using the CS-reconstructed signal. Therefore, it can be concluded that the data compression and the reconstruction using CS has not been carried out at the expense of the important information of the structural measurements. In other words, CS has successfully captured the important information of the original signal sufficient for damage detection and localization with the used damage detection methods.

Application of CS for an SHM system requires pre-knowledge of the sparsity of the signal. The measurement matrix has to be determined according to the sparsity and built-in to the sensors prior to installation. Any SHM system has to undergo some pre-testing phases before it is fully operational. Thus, in this pre-testing phase, the structural responses can be acquired using the intended sensors and tested for their sparsity. The measurement matrix can be determined accordingly. Once the sensors are installed and the SHM system is online, the signal can be acquired in an already compressed form during sampling itself, which gives the main advantage of saving sensor energy and memory space using CS for compressed data acquisition. These data are first stored in the sensors as usual, and then transmitted to the central server for processing and decision making.

The heavy computations such as convex optimization required for the reconstruction process are carried out at the central server where the compressed data are received. Thereby, 
CS offers another advantage by not placing a computational burden on the sensors. Using CS in an SHM system will not only provide very good reconstruction of the original structural response, impressive CRs and the above mentioned advantages, but also facilitates the system with the means of collecting the full signal at the central server. This will enable more studies and monitoring work to be carried out at convenience, without compromising the important information contained in the structural responses.

\section{Conclusions}

CS is a recent breakthrough in communication technology that introduces the means of reproducing a signal with a significantly lower number of acquired samples than the Nyquist's rate by exploiting the sparsity of a signal. Sampling the signal in an already compressed form, CS saves sensor memory space and power spent on data storage and compression processes. In this paper, CS is explored for its applicability in effective and efficient SHM using experimental structural response data from an RC structure. Using CS with SHM measurements, successful reconstruction of the signal was achieved with reconstruction accuracies as good as $99 \%$ at considerable compression levels. Since CR is a direct indication of energy and memory saving in the system, these results suggest that CS could be used for energy efficient and memory efficient SHM. Further analysis were carried out on the reconstructed signal for accuracy in structural damage identification. These analyses resulted in successful damage detection and localization in the structure using two structural damage identification methods: the ARD method and the Wiener filter method. Thus, it can be concluded that in this study, data compression through CS has retained the important information of the structural responses required for damage decision making. The success of this study is a good indication that CS has much potential in the development of efficient and effective SHM systems. 


\section{References}

[1] M. Crossbow, http://xbow.com (2013).

[2] Y. Bao, J. L. Beck, H. Li, Compressive sampling for accelerometer signals in structural health monitoring, Structural Health Monitoring 10 (3) (2011) 235-246.

[3] K.-Y. Wong, Y.-Q. Ni, Modular architecture of SHM system for cable-supported bridges, Encyclopedia of structural health monitoringdoi:10.1002/9780470061626.shm162.

[4] J. P. Lynch, A. Sundararajan, K. H. Law, A. S. Kiremidjian, E. Carryer, Embedding damage detection algorithms in a wireless sensing unit for operational power efficiency, Smart Materials and Structures 13 (4) (2004) 800.

[5] V. A. Kottapalli, A. S. Kiremidjian, J. P. Lynch, E. Carryer, T. W. Kenny, K. H. Law, Y. Lei, Twotiered wireless sensor network architecture for structural health monitoring, in: Smart Structures and Materials, International Society for Optics and Photonics, 2003, pp. 8-19.

[6] N. Xu, S. Rangwala, K. K. Chintalapudi, D. Ganesan, A. Broad, R. Govindan, D. Estrin, A wireless sensor network for structural monitoring, in: Proceedings of the 2nd international conference on Embedded networked sensor systems, ACM, 2004, pp. 13-24.

[7] E. J. Candes, M. B. Wakin, An introduction to compressive sampling, Signal Processing Magazine, IEEE 25 (2) (2008) 21-30.

[8] D. L. Donoho, Compressed sensing, Information Theory, IEEE Transactions on 52 (4) (2006) 1289-1306.

[9] W. Bajwa, J. Haupt, A. Sayeed, R. Nowak, Compressive wireless sensing, in: Proceedings of the 5th international conference on Information processing in sensor networks, ACM, 2006, pp. 134-142.

[10] C. Feng, S. Valaee, Z. Tan, Localization of wireless sensors using compressive sensing for manifold learning, in: Personal, Indoor and Mobile Radio Communications, 2009 IEEE 20th International Symposium on, IEEE, 2009, pp. 2715-2719.

[11] Q. Ling, Z. Tian, Decentralized sparse signal recovery for compressive sleeping wireless sensor networks, Signal Processing, IEEE Transactions on 58 (7) (2010) 3816-3827.

[12] M. Jayawardhana, X. Q. Zhu, R. Liyanapathirana, U. Gunawardana, Compressive sensing for structural damage detection of reinforced concrete structures, Key Engineering Materials 569 (2013) 742-750.

[13] M. Jayawardhana, X. Zhu, R. Liyanapathirana, Structural damage detection of RC structures using AR model coefficients, in: Proceedings of the 14th Asia Pacific Vibration Conference, December 2011, Hong Kong, 2011.

[14] M. Jayawardhana, X. Zhu, R. Liyanapathirana, Structural damage detection using the Wiener filter, 
in: Proceedings of the 22nd Australasian Conference on the Mechanics of Structures and Materials, December 2012, Sydney, Australia, 2012.

[15] M. Jayawardhana, X. Zhu, R. Liyanapathirana, Decentralized damage detection of RC structures using the Wiener filter, Australian Journal of Structural Engineering 14 (1) (2012) 57-69.

[16] J. Cortial, C. Farhat, L. J. Guibas, M. Rajashekhar, Compressed sensing and time-parallel reducedorder modeling for structural health monitoring using a dddas, in: Computational Science-ICCS 2007, Springer, 2007, pp. 1171-1179.

[17] Y. Bao, H. Li, J. L. Beck, J. Ou, Investigation of compressive sampling for structural vibration data, in: Proceedings of the 4th International Conference on Structural Health Monitoring of Intelligent Infrastructure, Empa-Akademie, 2009.

[18] Y. Bao, H. Li, X. Sun, Y. Yu, J. Ou, Compressive sampling-based data loss recovery for wireless sensor networks used in civil structural health monitoring, Structural Health Monitoring 12 (1) (2013) 78-95.

[19] Y. Wang, H. Hao, Damage identification scheme based on compressive sensing, Journal of Computing in Civil Engineering 29 (2) (2013) 1-10.

[20] D. Mascarenas, A. Cattaneo, J. Theiler, C. Farrar, Compressed sensing techniques for detecting damage in structures, Structural Health Monitoring 12 (4) (2013) 325-338.

[21] Y. Yang, S. Nagarajaiah, Structural damage identification via a combination of blind feature extraction and sparse representation classification, Mechanical Systems and Signal Processing 45 (1) (2014) 1-23.

[22] Y. Yang, S. Nagarajaiah, Blind denoising of structural vibration responses with outliers via principal component pursuit, Structural Control and Health Monitoring 21 (6) (2014) 962-978.

[23] Y. Yang, S. Nagarajaiah, Output-only modal identification by compressed sensing: Non-uniform lowrate random sampling, Mechanical Systems and Signal Processing 56-57 (2015) 15-34.

[24] H. Landau, Sampling, data transmission, and the nyquist rate, Proceedings of the IEEE 55 (10) (1967) $1701-1706$.

[25] X. Zhu, H. Hao, Damage detection of RC slabs using nonlinear vibration features, International Journal of Structural Stability and Dynamics 9 (04) (2009) 687-709.

[26] D. L. Donoho, De-noising by soft-thresholding, Information Theory, IEEE Transactions on 41 (3) (1995) 613-627.

[27] R. R. Coifman, D. L. Donoho, Translation-invariant de-noising, Springer, 1995.

[28] A. Antoniadis, G. Oppenheim, Wavelets and statistics, Springer-Verlag New York, 1995.

[29] D. L. Donoho, J. M. Johnstone, Ideal spatial adaptation by wavelet shrinkage, Biometrika 81 (3) (1994) 
$425-455$.

[30] A. Graps, An introduction to wavelets, Computational Science \& Engineering, IEEE 2 (2) (1995) 50-61.

[31] M. C. Grant, S. P. Boyd, Graph implementations for nonsmooth convex programs, in: Recent advances in learning and control, Springer, 2008, pp. 95-110.

[32] G. J. Pottie, W. J. Kaiser, Wireless integrated network sensors, Communications of the ACM 43 (5) (2000) 51-58.

[33] G. Anastasi, M. Conti, M. Di Francesco, A. Passarella, Energy conservation in wireless sensor networks: A survey, Ad Hoc Networks 7 (3) (2009) 537-568.

[34] K. Sayood, Introduction to data compression, Access Online via Elsevier, 2012.

[35] P. J. Brockwell, R. A. Davis, Introduction to time series and forecasting, Taylor \& Francis US, 2002.

[36] G. E. Box, G. M. Jenkins, G. C. Reinsel, Time series analysis: forecasting and control, Wiley. com, 2013.

Table 1: Comparison of CS performance against wavelet compression using Fixed and Huffman encoding.

\begin{tabular}{|c|c|c|c|c|c|c|c|c|}
\hline \multirow[t]{2}{*}{$\begin{array}{l}\text { Comparison } \\
\text { feature }\end{array}$} & \multicolumn{6}{|c|}{$\begin{array}{l}\text { Compressive sensing } \\
\text { ('rigrsure' threshold) }\end{array}$} & \multicolumn{2}{|c|}{$\begin{array}{c}\text { Wavelet } \\
\text { compression }\end{array}$} \\
\hline & $2 \times s$ & $3 \times s$ & $4 \times s$ & $5 \times s$ & $10 \times s$ & $15 \times s$ & Sym2 Lv2 & Db2 Lv10 \\
\hline Signal length & 220 & 330 & 440 & 550 & 1100 & 1650 & 2620 & 2070 \\
\hline $\mathrm{CR}$ & 18.64 & 12.42 & 9.32 & 7.45 & 3.73 & 2.48 & 1.56 & 1.98 \\
\hline $\begin{array}{l}\text { Reconstruction } \\
\text { accuracy }\left(R A_{C S}\right)\end{array}$ & 0.7025 & 0.8207 & 0.8600 & 0.8879 & 0.9777 & 0.9923 & 1.0000 & 1.0000 \\
\hline
\end{tabular}




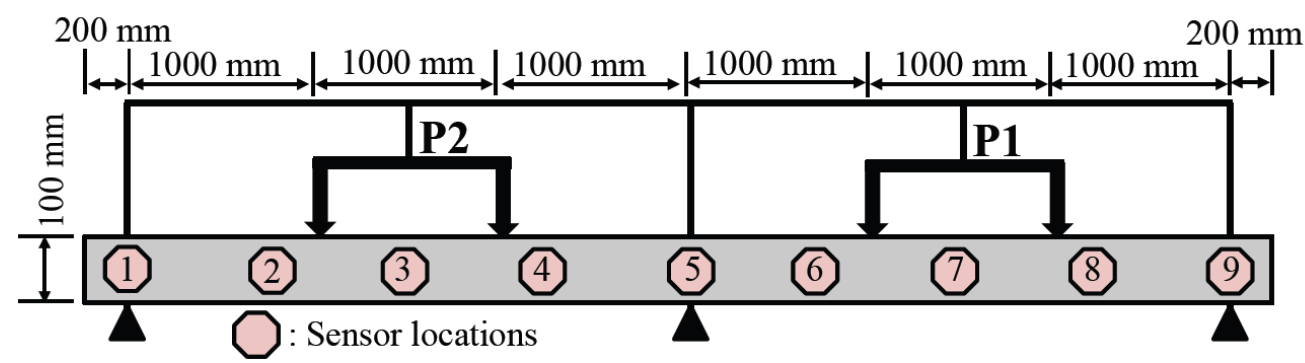

(a) Static test and sensor locations.

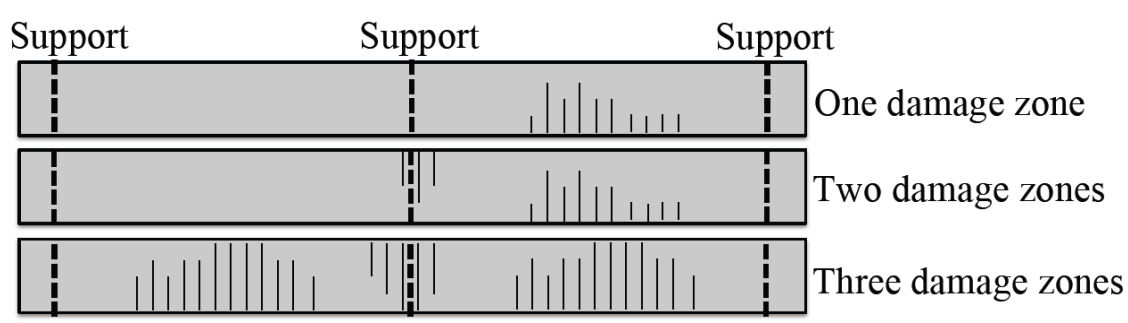

(b) Damage scenarios.

Figure 1: Experimental setup and damage scenarios.

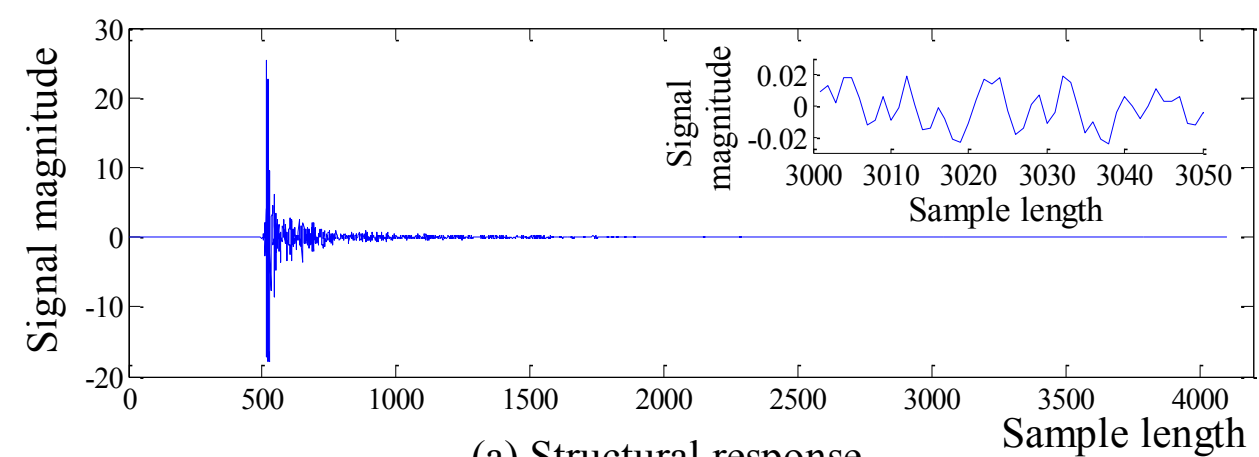

(a) Structural response

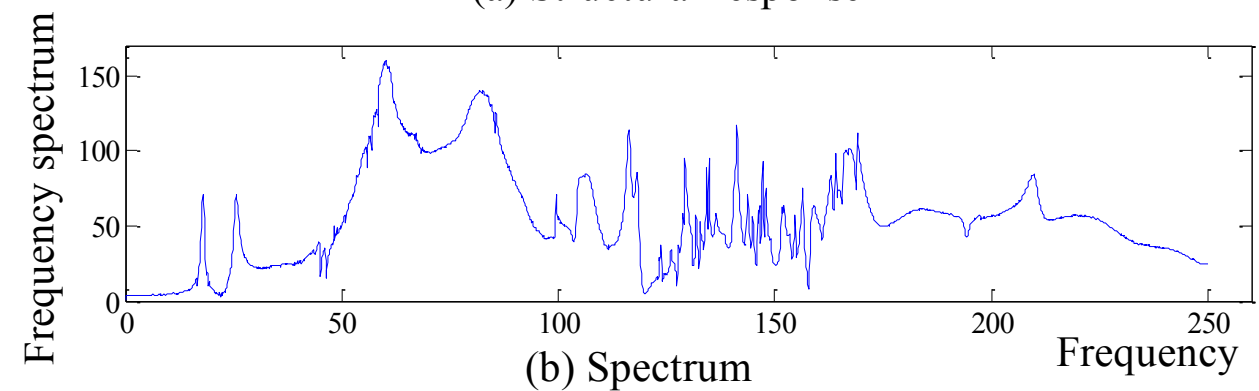

Figure 2: A sample of the structural impulse response and its frequency spectrum. 

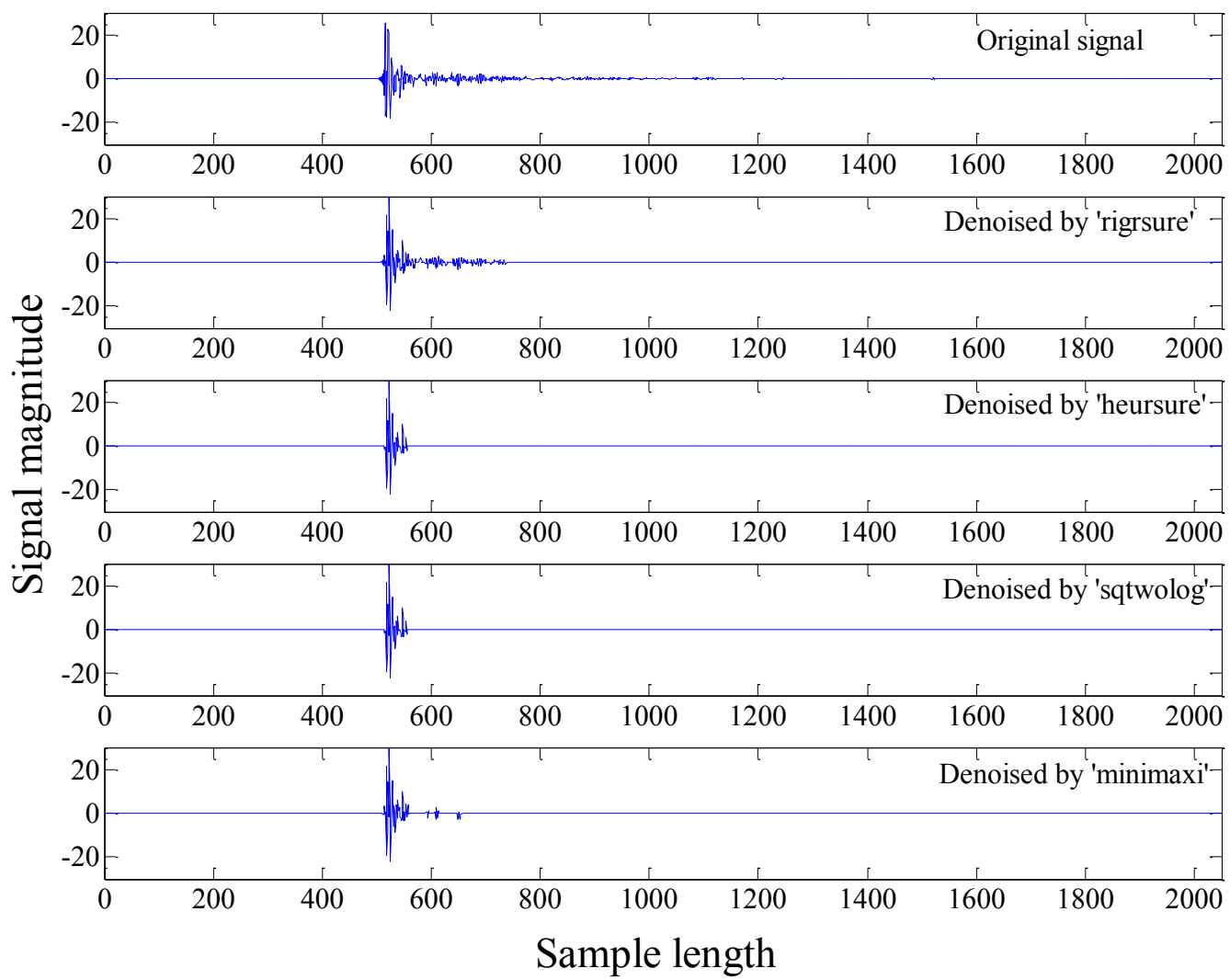

Figure 3: Original signal compared with the denoised signals using different threshold selections (Hard thresholded). 


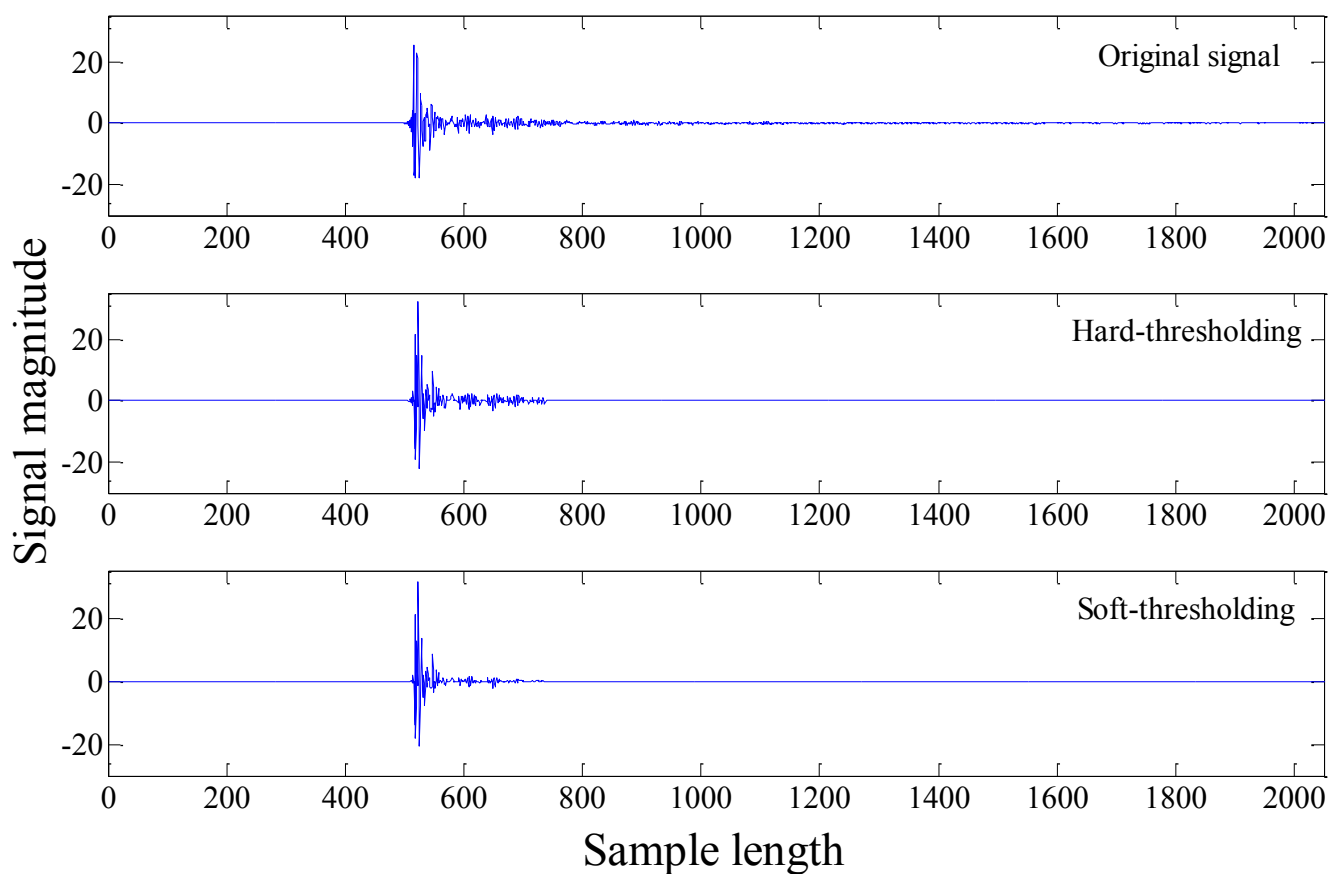

Figure 4: Original signal compared with the denoised signals using Hard and Soft thresholding (threshold selection using Stein's Unbiased Risk method). 


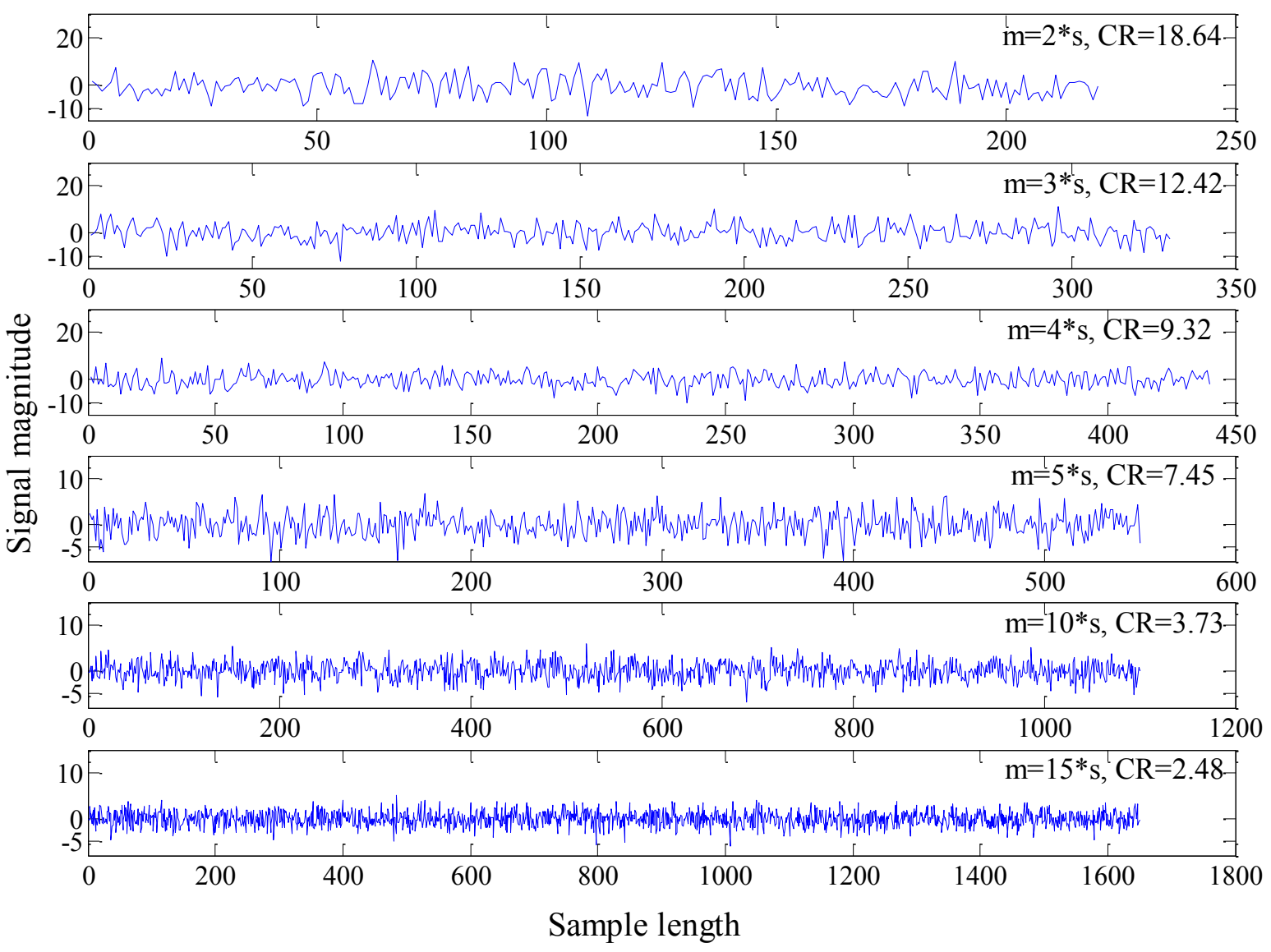

Figure 5: Compressed signals with different compression ratios. 

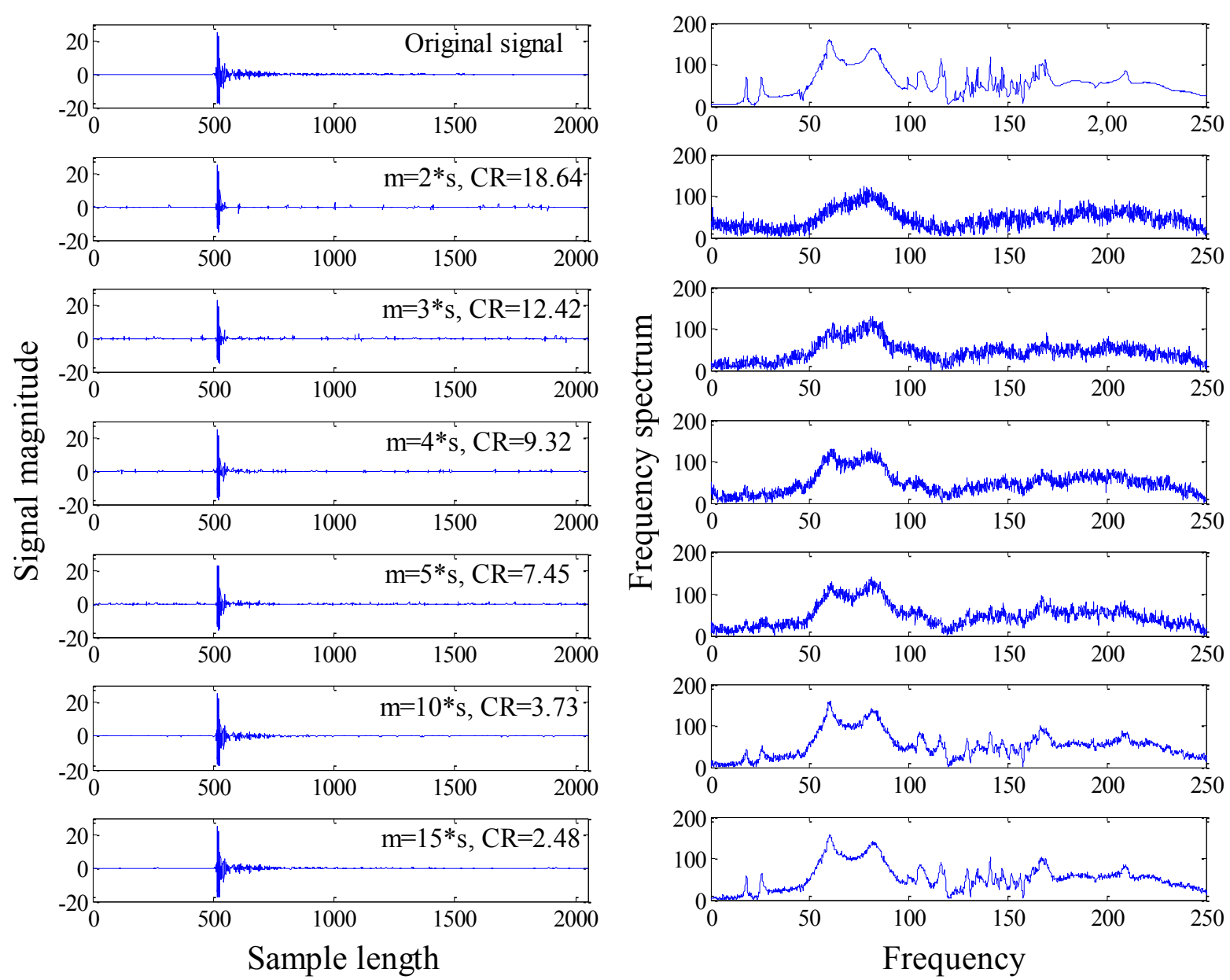

Figure 6: Reconstructed signals with different compression ratios, and their frequency spectra. 


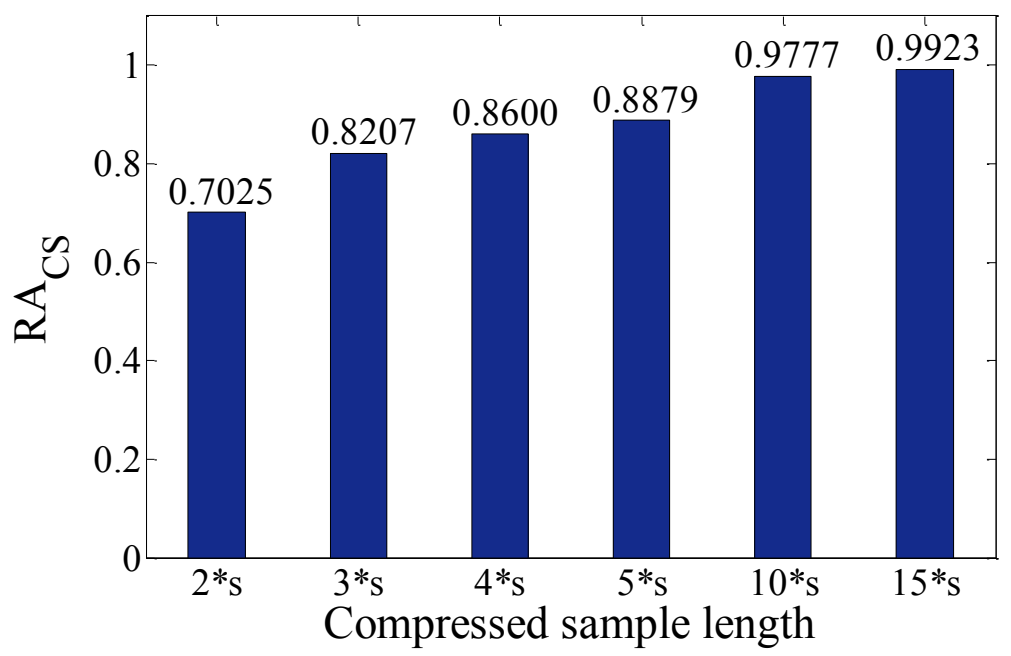

Figure 7: Reconstruction accuracy for different compression ratios.

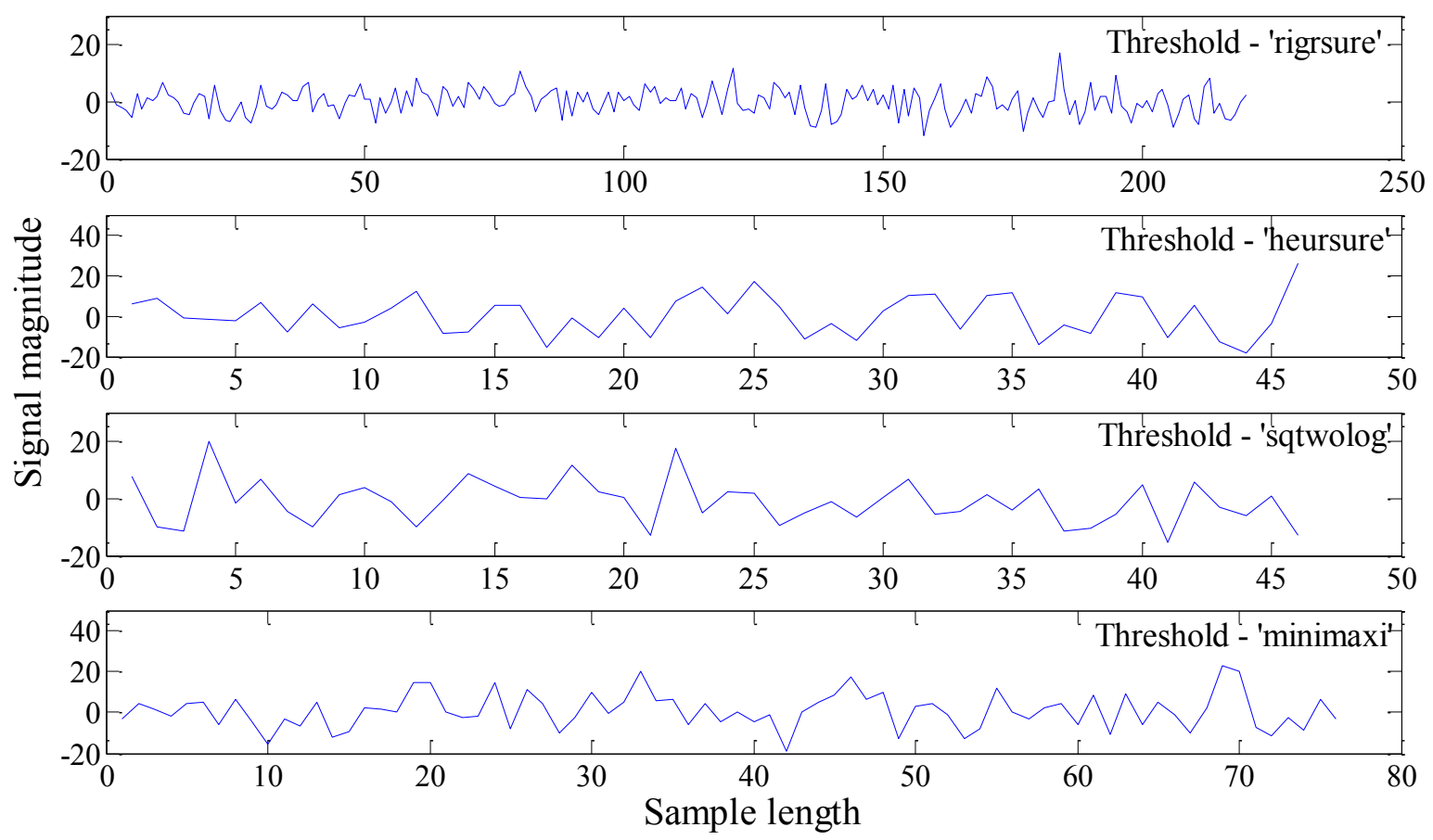

Figure 8: Compressed signals with different de-noising thresholds. 

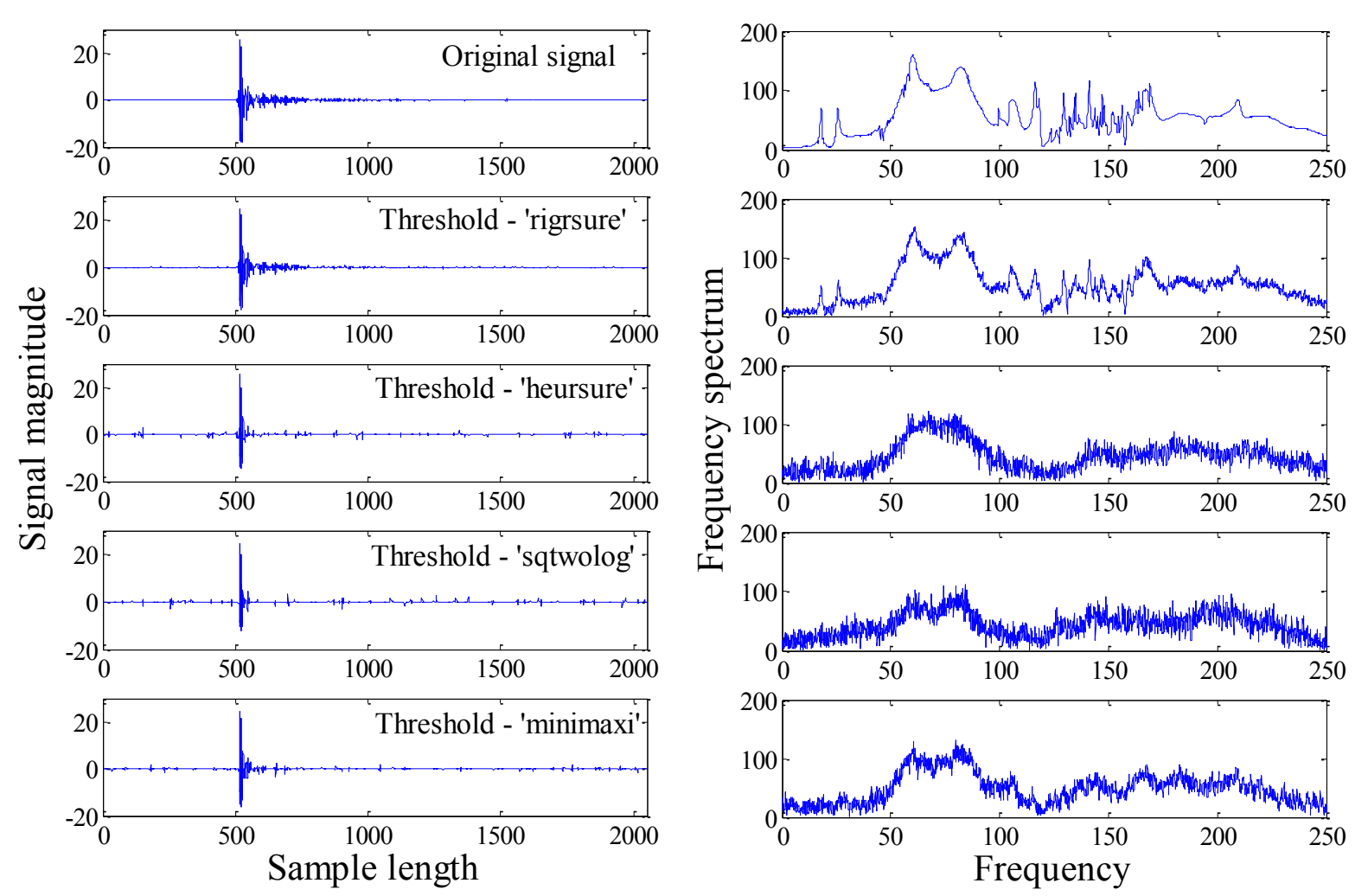

Figure 9: Reconstructed signals with different de-noising thresholds and their frequency spectra.

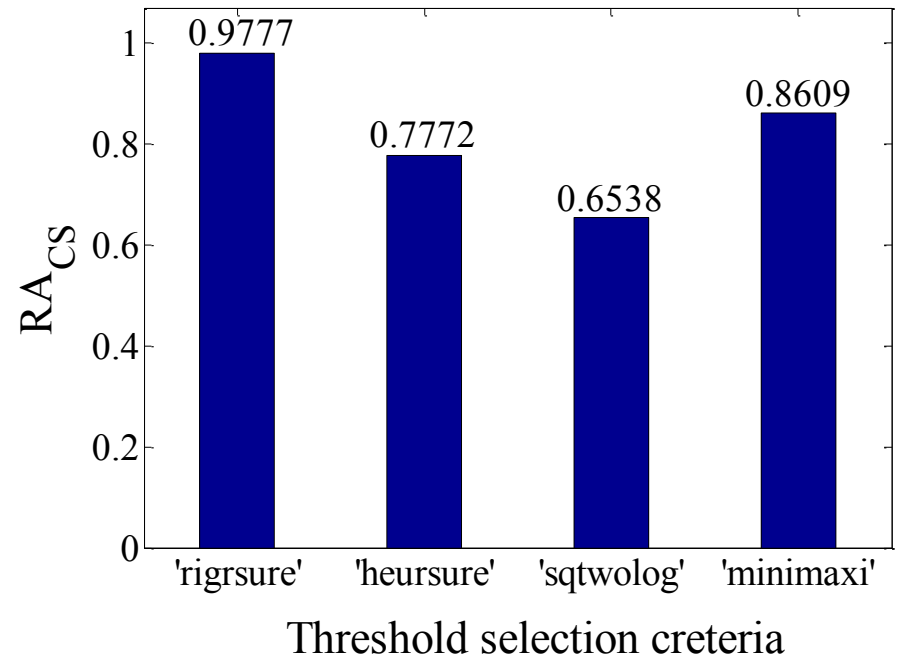

Figure 10: Reconstruction accuracy with different thresholding techniques. 


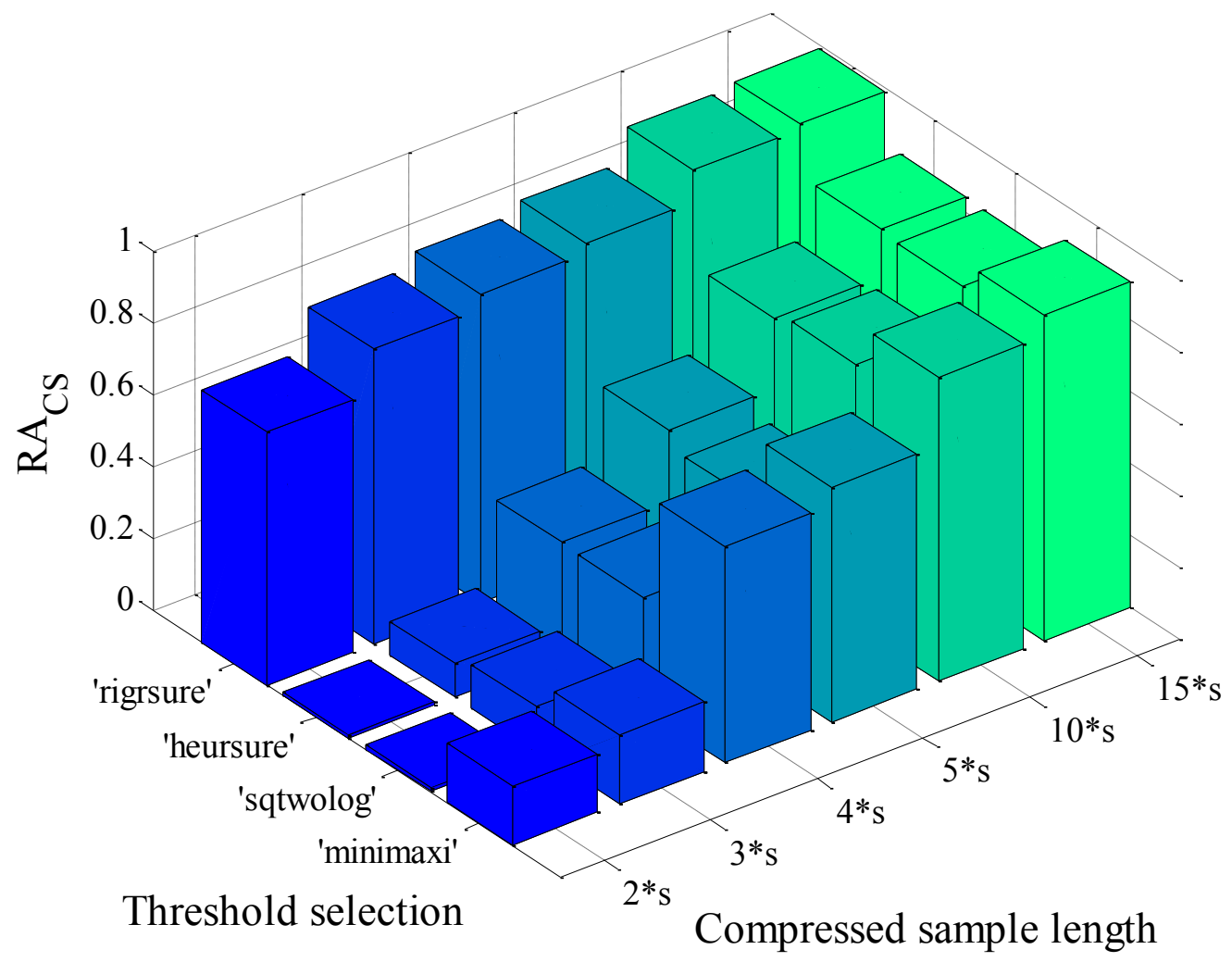

Figure 11: Reconstruction accuracy with different thresholding techniques and sample lengths.

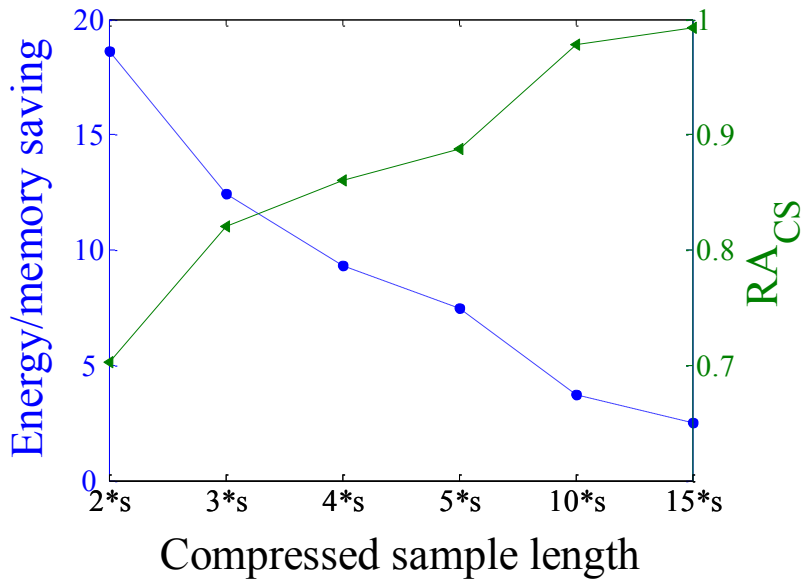

Figure 12: Sensor energy and memory saving with CS application. 

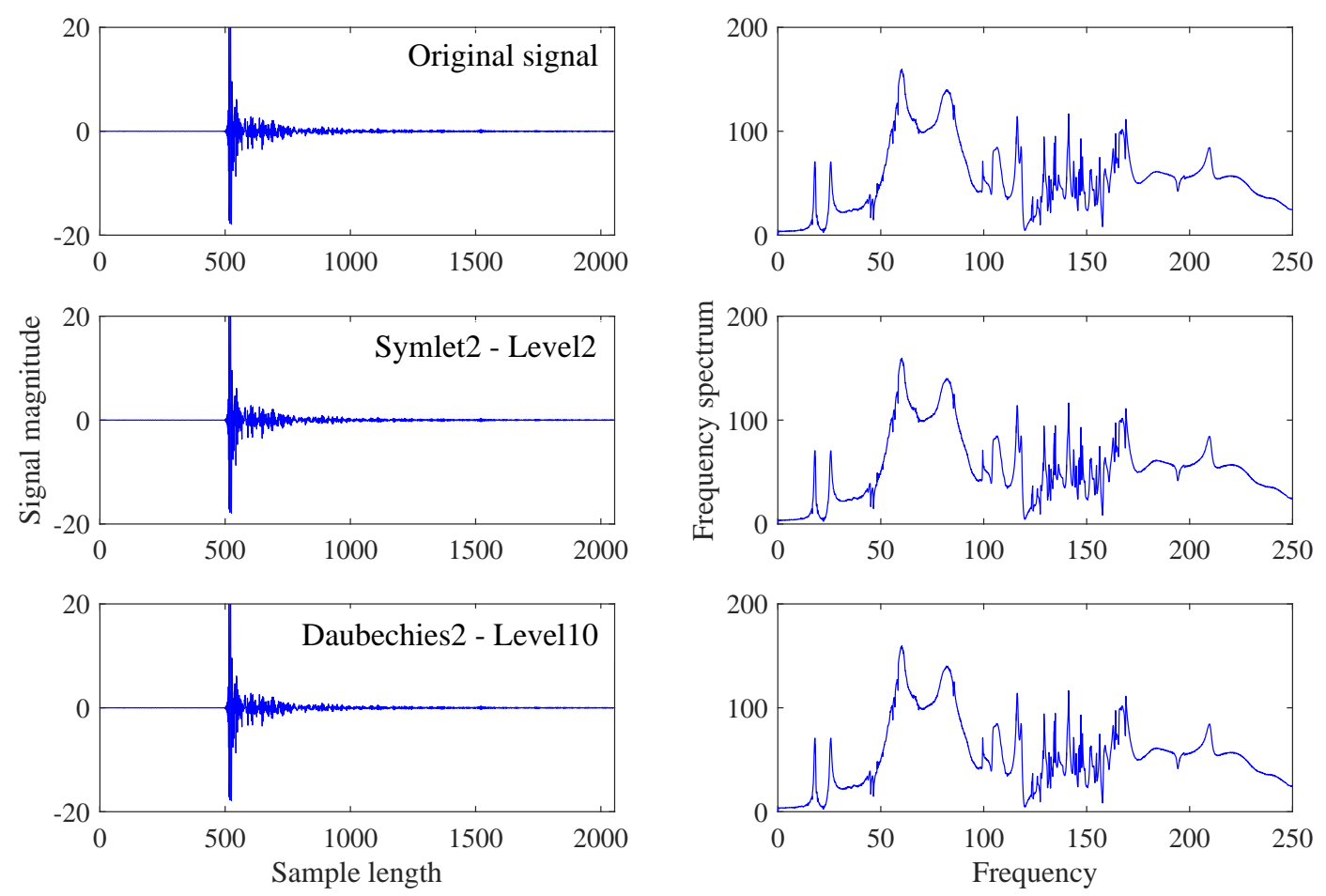

Figure 13: Reconstructed signals with different compression techniques and their frequency spectra.

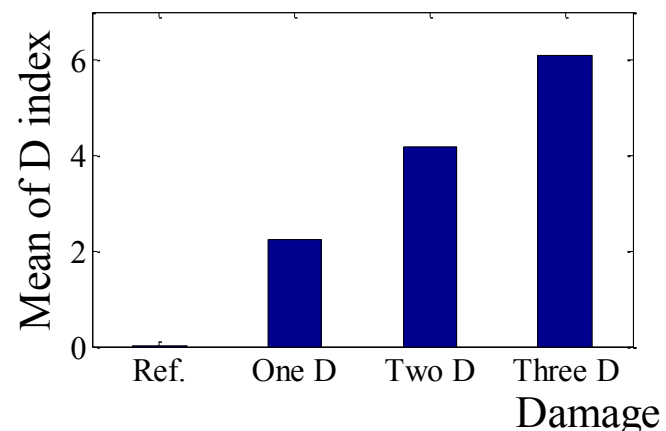

(a) Original data

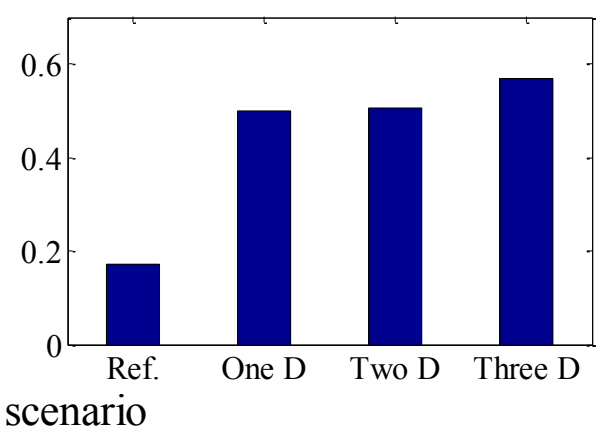

(b) CS-reconstructed data

Figure 14: Damage detection using the ARD method with Original data and CS-reconstructed data. 


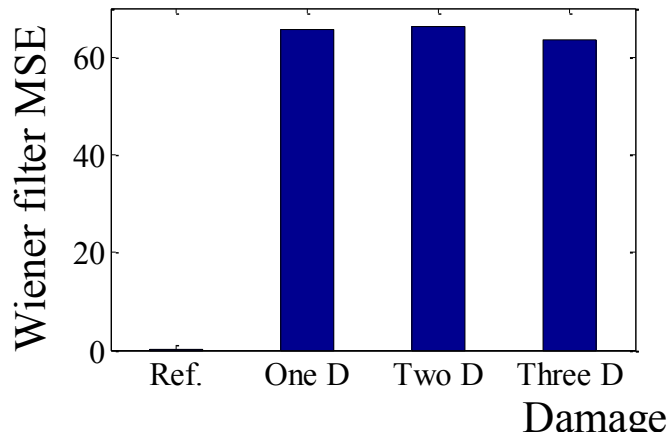

(a) Original data

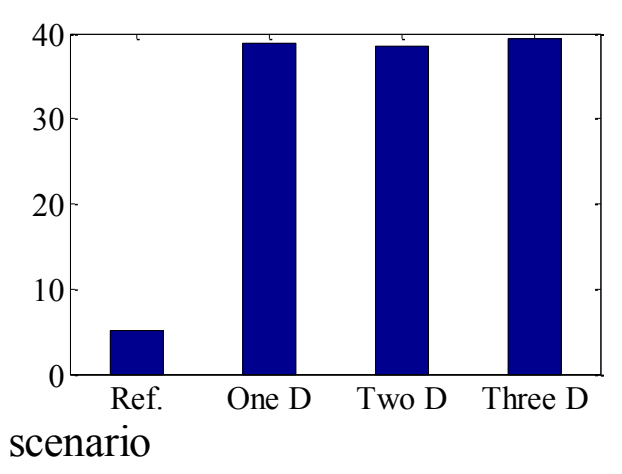

(b) CS-reconstructed data

Figure 15: Damage detection using the Wiener filter method with Original data and CS-reconstructed data.

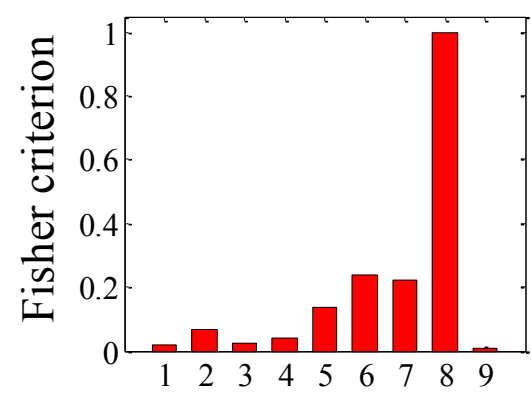

(a) One damage zone

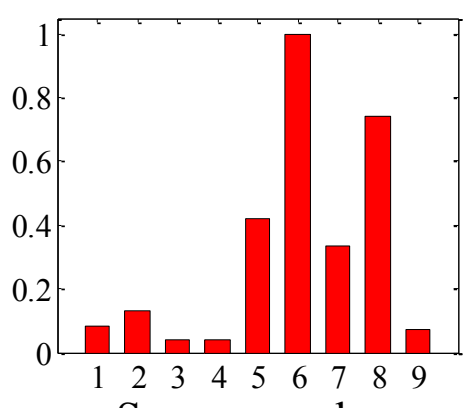

Sensor number

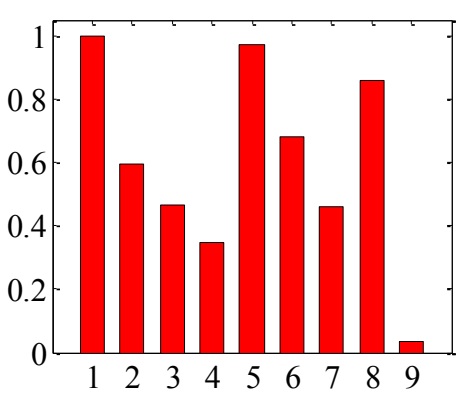

(c) Three damage zones

Figure 16: Damage localization using the ARD method.

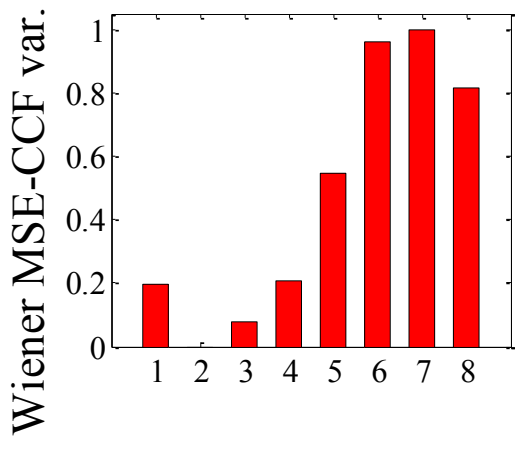

(a) One damage zone

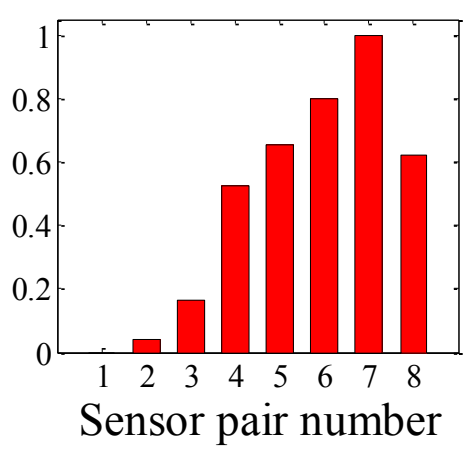

(b) Two damage zones

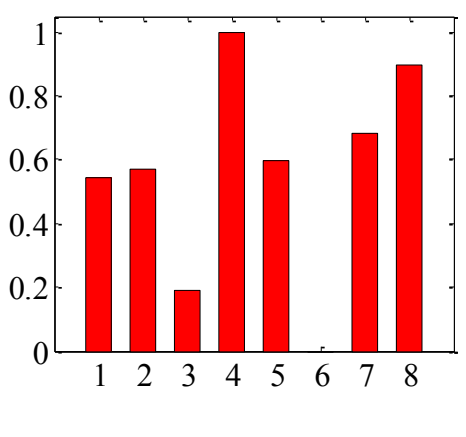

(c) Three damage zones

Figure 17: Damage localization using the Wiener filter method. 